\title{
Characterising the structural properties of polymer separators for lithium-ion batteries in 3D using phase contrast X-ray microscopy
}

Donal P. Finegan ${ }^{a}$, Samuel J. Cooper ${ }^{b}$, Bernhard Tjaden ${ }^{a}$, Oluwadamilola O. Taiwo ${ }^{a}$, Jeff Gelb ${ }^{c, d}$, Gareth Hinds ${ }^{\mathrm{e}}$, Dan J.L. Brett ${ }^{\mathrm{a}}$, Paul R. Shearing ${ }^{\mathrm{a} *}$

a. Electrochemical Innovation Lab, Department of Chemical Engineering, University College London, Torrington Place, London WC1E 7JE, UK

b. Earth Science and Engineering, Imperial College London, SW11 2AZ, UK

c. Department of Biomedical, Chemical, and Materials Engineering, San Jose State University, San Jose, CA, USA

d. Carl Zeiss X-ray Microscopy, Pleasanton, CA, USA

e. National Physical Laboratory, Hampton Road, Teddington, Middlesex TW11 OLW, UK

*Corresponding author: email p.shearing@ucl.ac.uk

\begin{abstract}
Separators are an integral component for optimising performance and safety of lithium-ion batteries; therefore, a clear understanding of how their microstructure affects cell performance and safety is crucial. Phase contrast X-ray microscopy is used here to capture the microstructures of commercial monolayer, tri-layer, and ceramic-coated lithium-ion battery polymer separators. Spatial variations in key structural parameters, including porosity, tortuosity factor and pore size distribution, are determined through the application of 3D quantification techniques and stereology. The architectures of individual layers in multi-layer membranes are characterised, revealing anisotropy in porosity, tortuosity factor and mean pore size of the three types of separator. Detailed structural properties of the individual layers of multi-layered membranes are then related with their expected effect on safety and rate capability of cells.
\end{abstract}

Key words: Lithium-ion batteries; separator; X-ray tomography; transport properties; characterisation; tortuosity.

\section{Introduction}

Balancing the performance and safety of lithium-ion batteries remains a challenge, in particular as lithium-ion batteries are becoming increasingly energy and power dense, as well as being required to operate reliably under a range of demanding conditions. The separator is an integral component in lithium-ion batteries, affecting both safety and performance [1-3]. Its primary function is to electrically isolate the positive and negative electrodes, whilst ensuring facile ionic transport of $\mathrm{Li}^{+}$ through the electrolyte; however, the design and selection of the separator can involve a trade-off between cell safety and charge/discharge rate, which may be optimised by design of the appropriate separator microstructure [4]. 
When considering the performance of a separator, there are three essential microstructural characteristics to take into account: porosity, tortuosity factor and pore size distribution (PSD). A high porosity, large mean pore size and low tortuosity contribute to a high rate capability, with low electrical impedance, commensurate with fast ion transport. However, such a structure is not effective in suppressing lithium dendrite growth between the electrodes, which can result in shortcircuiting. These important, and often conflicting, microstructural properties of separator membranes contribute to the overall performance and safety of a cell, and yet they remain poorly understood.

The microstructural properties of separator membranes are conventionally quantified through experimental methods; for example, mercury intrusion porosimetry (MIP) is most frequently used to measure porosity and PSD [5], and is integrated into standard testing methods such as ASTM D2873. However, this technique has a number of limitations [6-8], including the 'ink-bottle' or 'bottleneck' effect, where the intruding fluid enters a cavity at a pressure determined by the size of the cavity entrance, rather than the cavity itself. Consequently, MIP can bias the PSD towards smaller pore sizes, overestimating small pores and underestimating large pores. Additionally, MIP measurements are based on the Washburn equation $[9,10]$, which involves the simplified assumption of the pores having a cylindrical geometry, which is not the case for most separator membranes. For these reasons, MIP is generally viewed as an inadequate method for accurately determining the PSD of complex materials [7].

The tortuosity factor of the separator is most commonly determined via electrical conductivity measurements, and alternatively described in terms of porosity through the Bruggeman relationship $[5,11,12]$; however, this has known limitations [13]. Two frequently used metrics that reflect the transport resistance, and hence porosity and tortuosity factor of separator materials, are the MacMullin number [5, 14], which is defined as the ratio of the specific resistivity of the electrolyte to the specific resistivity of the separator soaked in electrolyte; and the Gurley number, which is a measure of the time required for a specific volume of air to pass through a separator under a certain pressure (former standard test ASTM-D726). Such characterisation methods have undergone little change since the advent of commercial lithium-ion batteries in the early 1990's [15], and do not adequately describe the microstructural properties of these materials. Only very recently have the transport properties of a polymer separator been characterised from a three-dimensional (3D) dataset captured using FIB-SEM tomography [16].

X-ray computed tomography (CT) is a powerful tool for characterising the microstructure of materials in 3D $[17,18]$ which has been successfully applied to materials within batteries [19-23] and other electrochemical energy devices [24-26]. Numerous studies have used X-ray CT to investigate the effect of the microstructural properties of electrode materials on the performance of lithium-ion batteries [20, 27-29], but few studies have focused on polymer separator materials, even though they are also expected to influence performance significantly. The low X-ray attenuation coefficients of polymer separators make segmentation of tomographic images challenging, and the fine microstructural features often found in commercial separator materials compound this. Here, we use the Zernike phase contrast technique applied to nano-scale X-ray microscopy [30], to capture and characterise the local microstructural properties of polymer separators in 3D. Three commercial separator samples are analysed: Celgard 2325 [31], Celgard 2500 [32], and the MTI ceramic-coated 
membrane [33], with tri-layer polypropylene-polyethylene-polypropylene (PP|PE|PP), monolayer $(\mathrm{PP})$, and monolayer (PE) coated with aluminium oxide, morphologies respectively [5].

For the first time, the porosity, tortuosity factor and PSD are determined locally through a combination of 3D quantification [6], stereological [34, 35], and image based modelling $[19,23]$ techniques, providing spatial information on the structural properties of commonly used commercial polymer separators.

\section{Materials and methods}

\subsection{Materials and properties}

This study focuses on three polymer separators; Celgard 2325 [31], Celgard 2500 [32], and the MTI ceramic-coated membrane [33]. Celgard 2325 consists of a tri-layer of PP|PE|PP, Celgard 2500 consists of a monolayer membrane of PP, and the MTI ceramic-coated membrane consists of a PE membrane coated on both sides with aluminium oxide particles. The manufacturer's specifications (shown in Table 1) state that the porosity of the Celgard 2325, 2500 and the MTI membranes are $39 \%, 55 \%$, and $37 \%$, respectively. This work is performed on dry separator membranes and does not account for the softening/swelling effects induced by the electrolyte.

Both Celgard separators are manufactured by dry processing, which is described in detail by Deimede and Elmasides [36]; this process involves uniaxial stretching of PP and PE lamellae, creating a highly directional 'slit-like' pore structure, which causes high anisotropy in the mechanical properties of the membranes. The MTI ceramic-coated separator is manufactured via wet processing, which involves biaxial stretching of a polymer film, resulting in low anisotropy in the mechanical properties of the membrane [36].

The porosity of the separator is defined as the ratio of void volume to apparent geometric volume. This can be approximately determined by weighing the sample before and after solvent is allowed to absorb into the pores. For a solvent of known density, the relative volume of absorbed solvent to the geometric volume of the separator can then be calculated. MIP [5] is also used to determine porosity according to the standard test method described in ASTM D-2873. In addition, MIP is used to determine the PSD and mean pore size of porous samples [5].

Table 1. Manufacturer specifications of separator material properties.

\begin{tabular}{llcccc}
\hline Sample & Type & $\begin{array}{c}\text { Thickness } \\
(\boldsymbol{\mu m})\end{array}$ & $\begin{array}{c}\text { Porosity } \\
\mathbf{( \% )}\end{array}$ & $\begin{array}{c}\text { Average PP Pore } \\
\text { Diameter } \\
(\boldsymbol{\mu m})\end{array}$ & $\begin{array}{c}\text { Gurley } \\
\text { number } \\
(\mathbf{s})\end{array}$ \\
\hline Celgard 2325 & Tri-layer & 25 & 39 & 0.028 & 620 \\
Celgard 2500 & Monolayer & 25 & 55 & 0.064 & 200 \\
MTI ceramic & Ceramic-coated & 16 & 37 & - & 240 \\
& PE monolayer & & & & \\
\hline \hline
\end{tabular}

The Gurley number is a measure of the resistance of the separator's architecture to the flow of air, and is the most frequently cited metric for mass transport through the membrane. The Gurley number is dependent on the flow regime of air through the porous network and it is not trivial to 
determine the tortuosity factor of a membrane from this value alone since Darcy's Law is only applicable for a low Reynolds number, which in most cases is not precisely known $[37,38]$. The influence of a separator on battery performance is more accurately measured by the ionic resistance $\left(R_{m}\right)$ of the membrane [39], which is a function of the electrolyte resistivity $\left(\rho_{e}\right)$, the tortuosity factor $(\tau)$, thickness of the membrane $(I)$, porosity $(\varepsilon)$, and membrane area $(A)$, according to:

$$
R_{m}=\rho_{e} \frac{\tau l}{\varepsilon A}
$$

\subsection{Imaging and segmentation}

The separator samples were imaged using a Zernike phase contrast setting in a lab-based nano-scale X-ray microscope (Zeiss Xradia 810 Ultra, Carl Zeiss Microscopy, Pleasanton, CA), which emphasizes material edges by using phase shifts across the incident X-ray beam [30, 40]. Fig. 1 shows an illustration of the principle behind the Zernike phase contrast technique employed here. Following transmission through the sample, the cone beam is focused using a zone plate. A phase ring then induces a known phase shift and amplitude reduction of the non-diffracted light. The diffracted and non-diffracted light are now of similar intensity but different phase and so interact to form a high contrast image on the detector plane. This is particularly useful for weakly attenuating materials such as polymers. In this study, a $5.4 \mathrm{keV}$ quasi-monochromatic beam was used and images were recorded on a $1024 \times 1024$ pixel charge-coupled device $(C C D)$ detector.

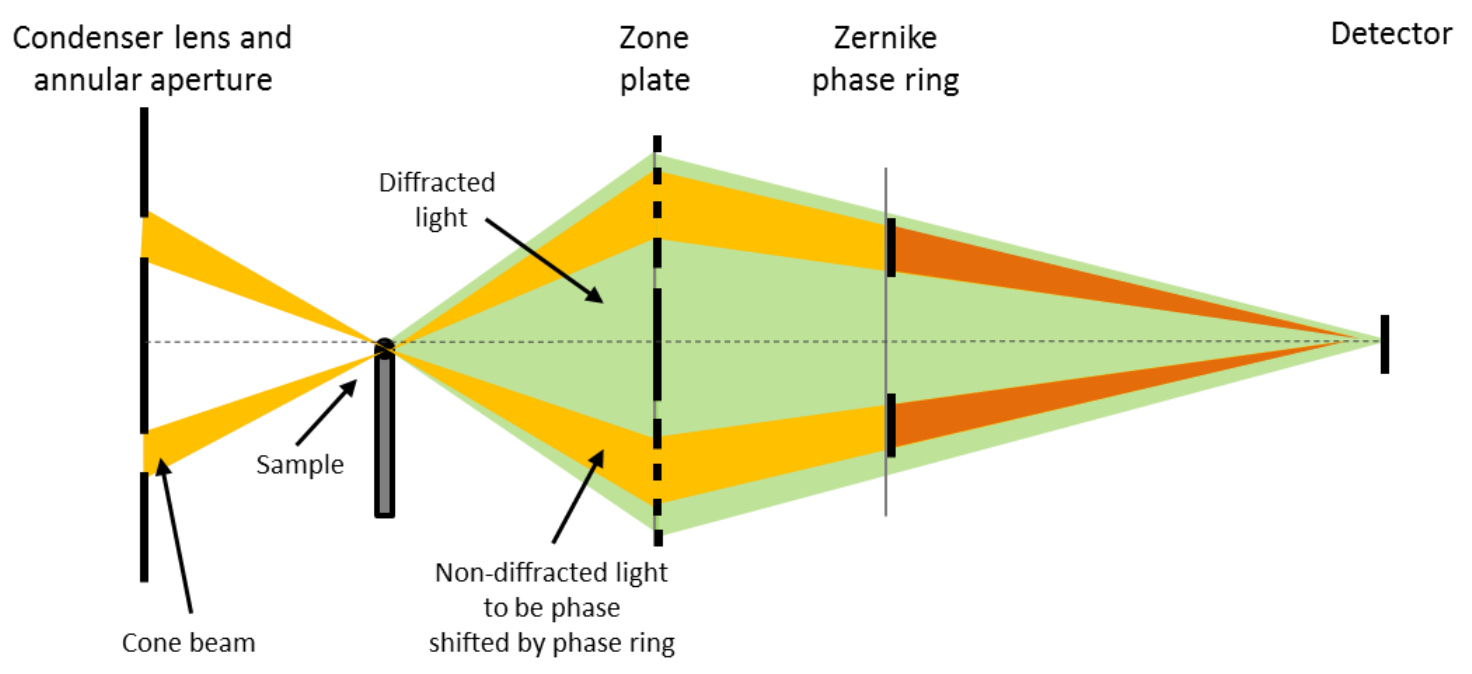

Fig. 1. Schematic showing the principle of the employed Zernike phase contrast technique.

A total of 2001 and $1751 \mathrm{X}$-ray transmission radiographs were captured for the Celgard 2325 and Celgard 2500 samples respectively, over $180^{\circ}$ rotation. The exposure time per radiograph for both samples was $35 \mathrm{~s}$, using a detector pixel size of $63.1 \mathrm{~nm}$. For the MTI ceramic-coated separator, 1601 images with a $15 \mathrm{~s}$ exposure time over a $180^{\circ}$ rotation were used to reconstruct the tomograms, and a detector binning setting of 2 yielded an effective voxel size of $126.2 \mathrm{~nm}$. Detector binning settings of 1 and 2 showed little difference in the effective resolution of the phase contrast images. The transmission images from both scans were reconstructed in 3D using ZEISS XMReconstructor software, which uses a filtered back-projection algorithm. Although high energy X-rays are known to 
damage polymer materials by inducing cross-linking of polymer chains [41, 42], any significant change in the polymer microstructure resulting from the damage during imaging could be seen via motion artefacts and poor image quality in the reconstructed tomograms. The reconstructed images showed clear edge definition, and no signs of motion artefacts, therefore any damage to the polymer material are thought to have a negligible effect on the separators microstructure.

Volume visualisation and image processing were performed using the Avizo software package (version 9, FEI, VSG). The polymer phase was segmented based on its higher grey-scale value and saved as a binary TIFF file. The pore volume was separated from the solid phase using threshold segmentation. When compared to the grey-scale images, the features in segmented images correlated well. Grey-scale images and the segmented binary images can be compared in Fig. 2, and further examples for comparison are provided in Supplementary Information. The samples were oriented such that the surface of the separator was perpendicular to the Z-axis (through-plane direction). In the $X, Y$ and $Z$ directions, the samples were cropped to volumes consisting of $822 \times 961$ $\times 415$ voxels $(51.87 \mu \mathrm{m} \times 60.65 \mu \mathrm{m} \times 25.19 \mu \mathrm{m})$ for Celgard 2325, $672 \times 654 \times 409$ voxels $(42.41 \mu \mathrm{m}$ $\times 41.27 \mu \mathrm{m} \times 25.81 \mu \mathrm{m})$ for Celgard 2500, and $379 \times 465 \times 138$ voxels $(47.75 \mu \mathrm{m} \times 58.59 \mu \mathrm{m} \times 17.39$ $\mu \mathrm{m})$ for the MTI ceramic-coated separator. 3D image reconstructions and their associated grey-scale and binary slices of each sample were made, and the segmented 3D TIFF image files were then used for image-based quantification and characterisation.

\subsection{Quantifying pore size distribution}

Two methods were used to determine the PSD: the first was a continuous PSD (c-PSD) 3D method developed by Munch and Holzer [6], the principle of which is based on expanding spheres from points along calculated centroid paths. Munch and Holzer provide the algorithm as an open source plugin for the Fiji image processing package [6]. In this method, a distance map is determined whereby voxels are assigned a value corresponding to the shortest distance to the phase boundary, from which the volume fraction of the pore phase that can be filled with spheres of a certain radius may be calculated. The c-PSD algorithm then plots the radii of spheres (increasing in radius by steps of $10 \mathrm{~nm}$ ) against the corresponding filled volumes, outputting a cumulative PSD plot of pore size as a function of volume fraction of the total pores.

The second method is based on a stereological approach where the mean pore radius, $L$, within individual slices in the $X, Y$ and $Z$ directions, is determined by calculating the mean volume to surface ratio according to Equation 2, where $V_{v}$ is the volume fraction of the pore, and $S_{v}$ is the interface density $[34,35]$.

$$
L=2 \frac{V_{v}}{S_{v}}
$$

This approach was implemented in MATLAB on a slice-by-slice basis, where the interfacial area was calculated by first identifying solid phase pixels that are connected to the pore phase, and then labelling each pixel with the number of neighbours of the opposite phase (i.e. 1-4). The boundary length is determined by summing the values of the labelled boundary pixels. For each separator specimen, separate PSD's are presented as normalised frequencies of the mean pore size of slices. 
Stereology-based PSD's were calculated for each of the axial directions, demonstrating pore size anisotropy. When compared to 3D quantification techniques, stereological methods tend not to be as accurate for measuring bulk parameters [43], but are useful for identifying deviations in porosity and pore size between, and along, different axial directions.

\subsection{Tortuosity factor measurement}

Most analyses of the tortuosity factor of separator materials involve electrical conductivity measurements [11, 44, 45], where the ratio of the resistivity of the electrolyte-filled membrane, to that of the electrolyte alone, is defined as:

$$
\frac{\rho_{s}}{\rho_{e}}=\frac{\tau}{\varepsilon}
$$

where $\rho_{s}$ is the resistivity of the membrane soaked in electrolyte, and $\rho_{e}$ is the resistivity of the electrolyte. This is a highly effective method for estimating the tortuosity factor of membranes, but does not provide local microstructural information, which is important for determining regions that most restrict ionic transport, particularly for multi-layer separators [46].

In this study, the tortuosity factors were calculated using a finite difference simulation, where the segmented voxel data were directly used as the simulation grid to avoid any potential smoothing effects from re-meshing. The steady-state scalar diffusion equation was solved with a fixed potential difference applied at two opposite faces of the volume (in the direction of the $Z$ axis), similar to the approach described by Cooper et al. [19]. By comparing, the simulated flow rate through the pore network, $Q_{\text {pore }}$, to that calculated for a fully porous control volume with the same outer dimensions, $Q_{c v}$, the tortuosity factor in a specific direction can be determined from the following expression:

$$
\tau=\varepsilon \frac{Q_{C V}}{Q_{\text {pore }}}
$$

Due to the large number of nodes in this simulation ( $>10^{8}$ voxels), an iterative over-relaxation method was employed to accelerate convergence. The code, TauFactor [47, 48], developed by Cooper, was implemented in MATLAB and is freely available online.

\section{Results and discussion}

\subsection{Porosity}

3D image reconstructions of each sample and associated grey-scale and binary slices are shown in Fig. 2. The porosity of the binary images of the full volumes was $41 \%$ for Celgard $2325,53 \%$ for Celgard 2500, and 38\% for the MTI ceramic-coated separator. 


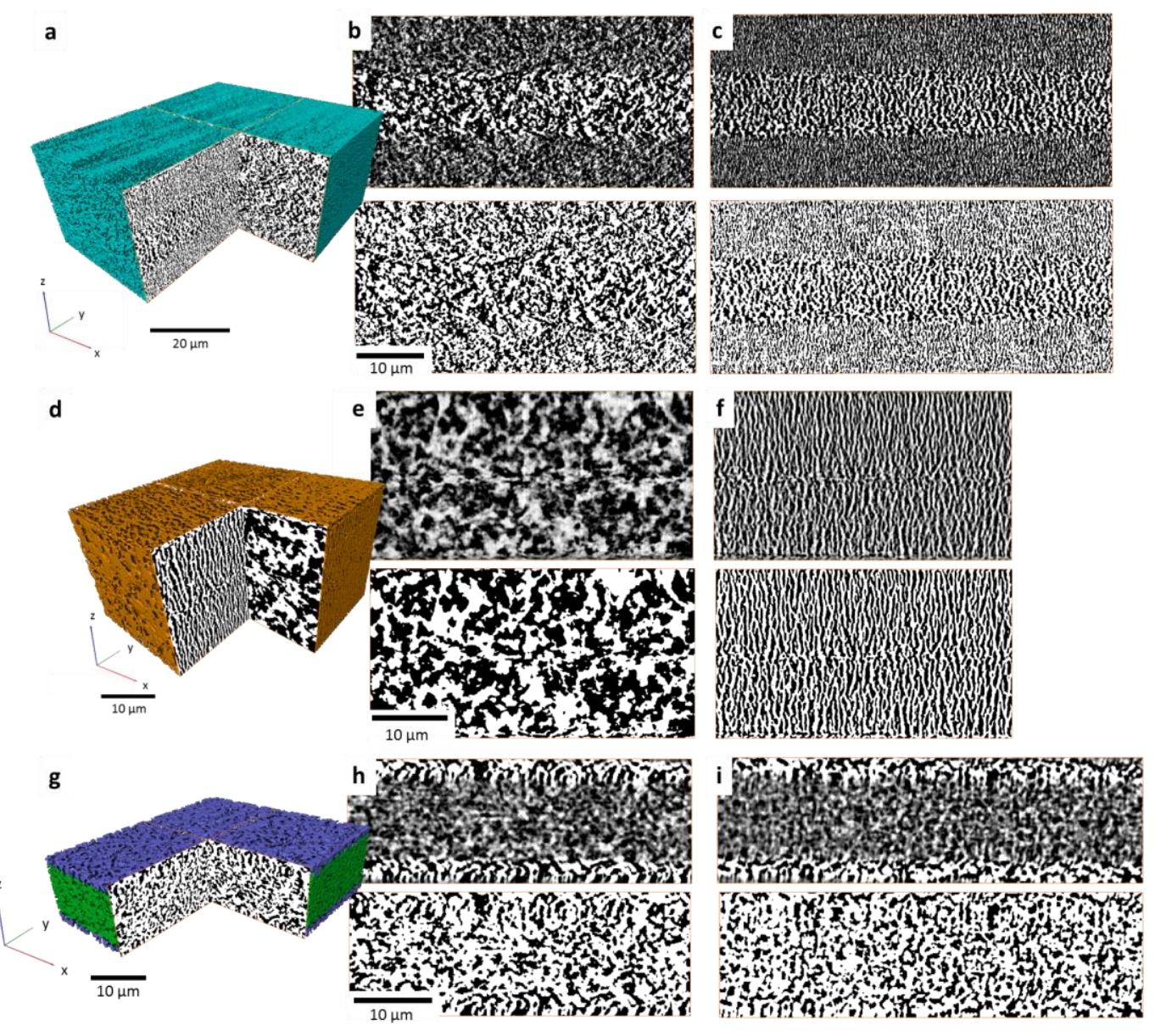

Fig. 2. a) Volume rendering of Celgard 2325 with binary slices in the $Y Z$ and $X Z$ planes. b) Grey-scale (top) and binary (bottom) slices from the $X Z$ plane and c) $Y Z$ plane showing clearly the tri-layer structure (PP|PE|PP). d) Volume rendering of Celgard 2500 with binary slices in $Y Z$ and $X Z$ planes. e ) Grey-scale (top) and binary (bottom) slices from the $X Z$ plane, and f) $Y Z$ plane showing the monolayer structure. g) Volume rendering of the MTI ceramic-coated membrane showing ceramic (blue) and PE (green) layers. h) Grey-scale (top) and binary (bottom) slices from the $X Z$ plane and i) $Y Z$ plane. In the binary images, white is solid and black is pore. The difference in pore structures observed in the $Y Z$ and $X Z$ planes highlight that the pores are elongated, caused by the extrusion process during manufacturing.

The porosity was also calculated on a slice-by-slice basis, where each slice is a single voxel thick (63.1 nm for Celgard samples and $126.2 \mathrm{~nm}$ for the MTI sample), to demonstrate that the porosity in the separator samples is non-uniform and anisotropic. A representative volume analysis of the porosity of the separators (provided in Supplementary Information) demonstrates that the samples are sufficiently large to be considered as being representative of the bulk material. Fig. 3a-c shows the slice-wise plots of porosity in the $X, Y$ and $Z$ directions. In the $Z$ direction, the tri-layer Celgard 2325 shows a significant difference in porosity with depth, revealing the PP layers to have a porosity of $c a .39 \%$, and the intermediate PE layer to have a porosity of $c a .44 \%$. The monolayer Celgard 2500 shows a drop in porosity of $c a$. 3\% mid-way through the separator. Upon close inspection, this region can also be seen mid-way through the sample in Fig. 2e, and may be an artefact of applying two layers of PP during the manufacturing process, with the region surrounding the interface having an anomalous porosity. For the MTI ceramic-coated separator, a higher porosity is observed for the ceramic coating than for the PE membrane. The porosities in the $X$ and $Y$ directions (Fig. 3b,c) fluctuate evenly around the mean for each sample. 

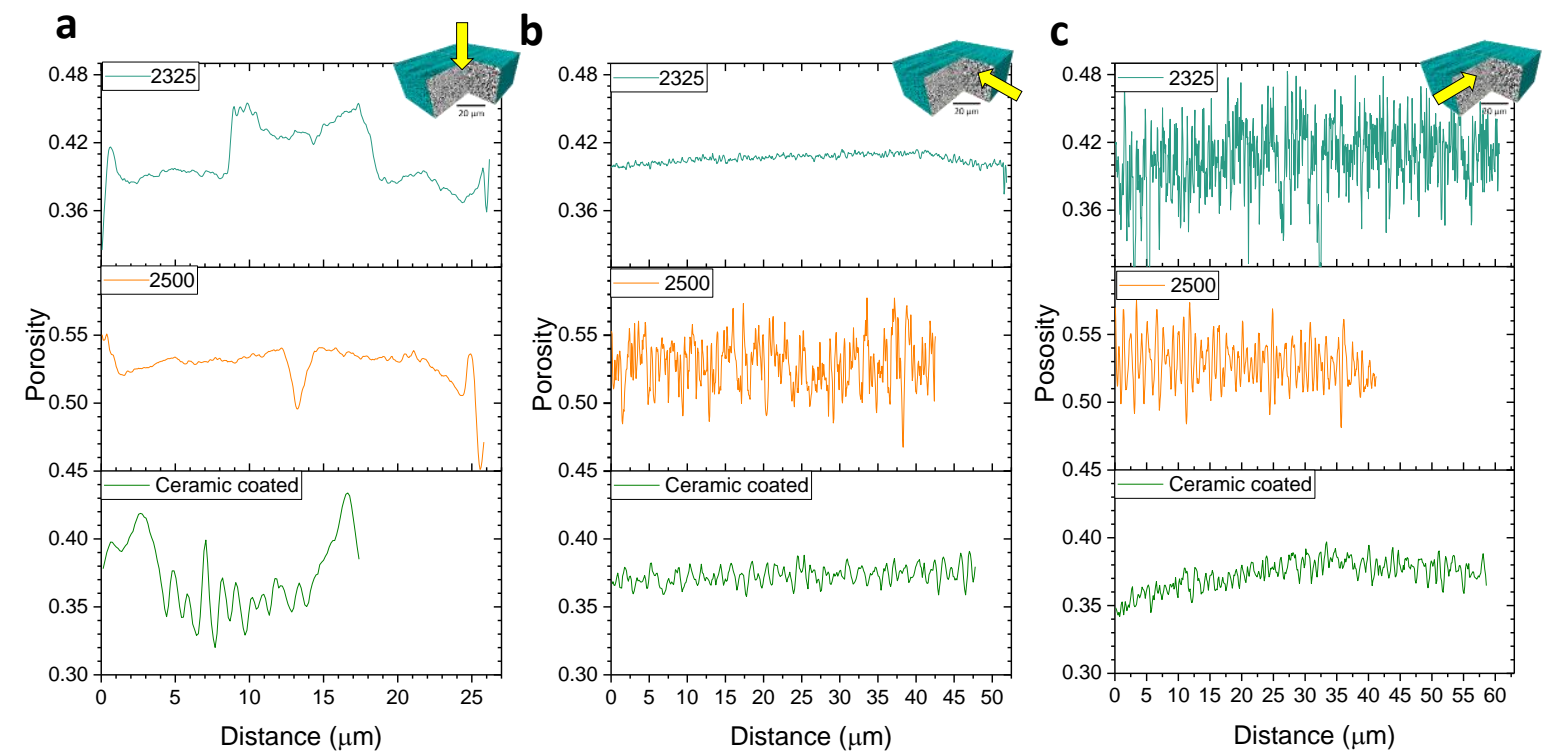

Fig. 3. a) Slice-wise porosity along the Z-axis for Celgard 2325 (top), 2500 (middle) and the MTI ceramic-coated separator (bottom). b) slice-wise porosity along the $X$-axis, and c) slice-wise porosity along the $Y$-axis.

\subsection{Pore size distribution}

Cumulative PSD plots of volume fraction against pore radius, determined by the c-PSD method described in Section 2.3, are shown in Fig. 4. The majority of the pore volume consists of pores of radii $70 \mathrm{~nm}-150 \mathrm{~nm}$ for Celgard 2325, $200 \mathrm{~nm}-300 \mathrm{~nm}$ for Celgard 2500, and $120 \mathrm{~nm}-320 \mathrm{~nm}$ for the MTI ceramic-coated separator. The predominance of pores near the resolution limit for Celgard 2325 and the MTI ceramic-coated membrane suggests the quantification of PSD may be limited by the resolution of the scan.

Average pore sizes calculated from the PSD plots in Fig. 4 are significantly larger than those specified by the manufacturer, with pore diameters that are greater by a factor of between 5 and 10 for Celgard 2325 and 2500. For Celgard 2325, the inclusion of the PE phase, which is shown to have a PSD consisting of much larger pores than the PP phase, would contribute to the much larger pore size measurement observed via the c-PSD method. The manufacturer specifies that the average pore size of the Celgard 2325 is based on the PP phase only, which is perhaps due to limitations of the utilised PSD method such as the 'ink-bottle' effect. Further study on the constrictivity of pores [26] is needed to provide a quantitative explanation for the deviation between experimental measurements and image-based geometric measurements presented here. Additionally, the X-ray microscopy imaging method in this study is limited by a pixel resolution of $63.1 \mathrm{~nm}$ for the Celgard samples, and $126.2 \mathrm{~nm}$ for the ceramic-coated sample; any pores smaller than this would not be detected and are therefore neglected in the PSD calculation. Structural features such as nano-fibrils that stretch across the slit-like pore openings (these are clearly seen in the publication by Arora and Zhang [5], and further SEM images of the surfaces of the membranes analysed here provided in Supplementary Information) are also not detected, and would certainly reduce the sphere radii capable of fitting into the pore phase. 


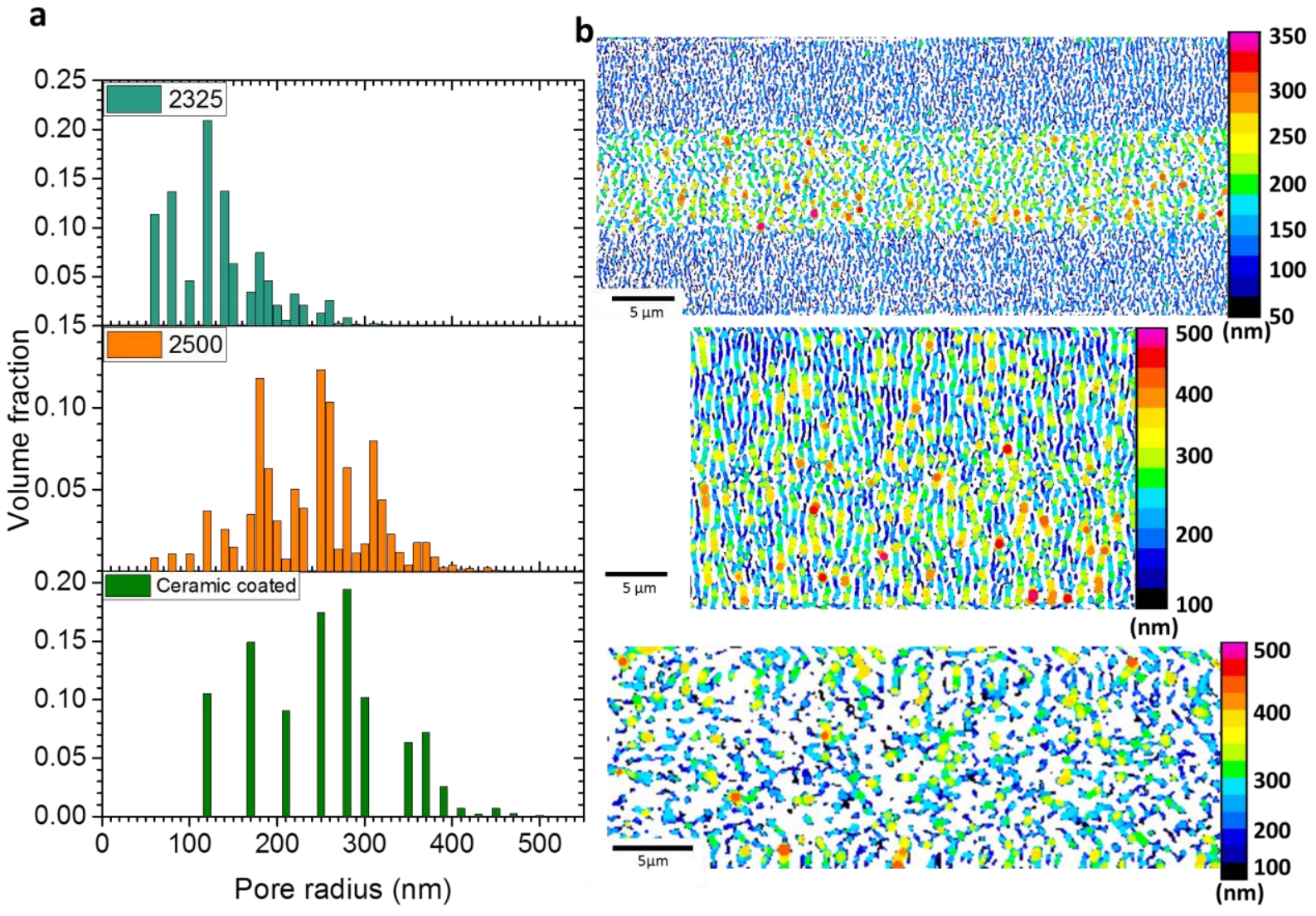

Fig. 4. a) PSD plots for Celgard 2325 (top), Celgard 2500 (middle) and the MTI ceramic-coated separator (bottom) based on the c-PSD method developed by Munch and Holzer [4]. b) Corresponding 2D slices from the YZ plane for Celgard 2325 (top), Celgard 2500 (middle) and the MTI ceramic-coated separator (bottom), where the radius of the largest possible sphere to fit in the pore, centred on the centroid path through the network, is colour-coded. The colour-coded pore structure within the Celgard 2325 (top) tri-layer membrane (PP|PE|PP) shows the intermediate PE layer to contain the largest pores.

The stereological approach $[34,35]$ was used to determine the PSD from slice-by-slice calculations of the mean pore size in each of the axial directions, to more closely examine the anisotropic and nonuniform properties of the pore structure of the separators (Fig. 5). In the $Z$ (through-plane) direction, the mean pore radius increases from $c a .60 \mathrm{~nm}$ to $c a .90 \mathrm{~nm}$ between the PP and PE layers for Celgard 2325 (Fig. 5a), which is consistent with what is observed using the c-PSD approach in Fig. 4b. An increase in pore size is identified in the $Z$ (through-plane) direction mid-way through Celgard 2500 (Fig. 5a). Fig. 5a also shows that the mean pore size for the ceramic layers in the MTI ceramiccoated membrane is higher than in the central PE layer. 

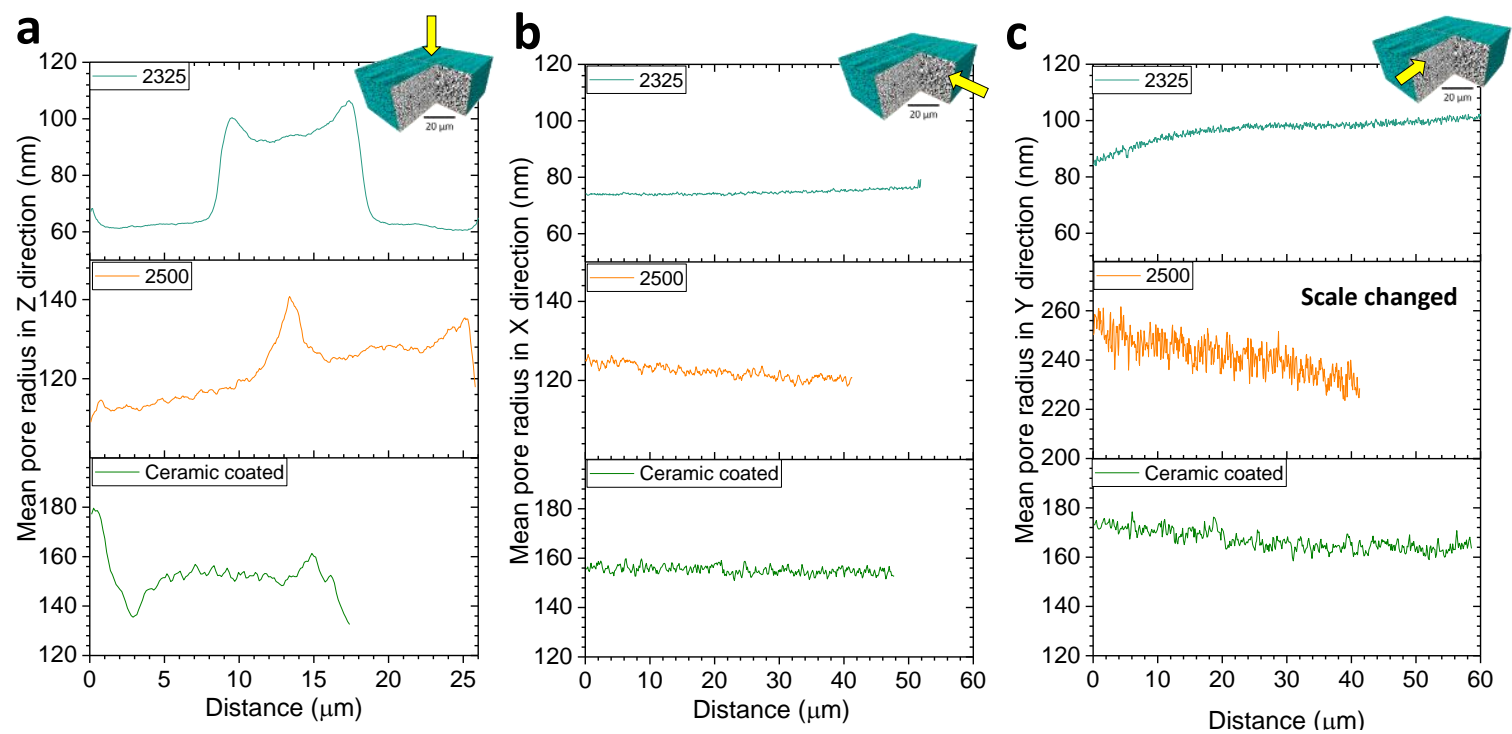

Fig. 5. Mean pore size, calculated via a stereological approach, for individual slices in the a) $Z$, b) $X$, and c) $Y$ directions for Celgard 2325 (top), Celgard 2500 (middle) and the MTI ceramic-coated separator (bottom). Note the change in the $Y$-axis scale for Celgard 2500 in the $Y$ direction.

Differences in mean pore size in the $X$ and $Y$ directions (Fig. 5b,c) for each sample demonstrate the extent of elongation of the pores. The manufacturing process of both Celgard membranes involves uniaxial stretching of polymer lamellae following extrusion and annealing [36], resulting in highlyoriented, deep, slit-like pore structures. This is particularly notable for Celgard 2500, where the mean pore size in the $Y$ direction is more than twice that in the $X$-direction. In contrast, the MTI ceramic-coated membrane is manufactured using a wet process, where biaxial stretching of a single extruded layer results in a relatively anisotropic mean pore diameter. In Fig. 6, the slice-wise pore size is presented as normalised frequency plots for each of the axial directions. The twin peaks in the Z-direction for the Celgard 2325 are caused by the region with relatively large pore size mid-way through the separator. There is a significant difference between the calculated pore size in the $X$ and $Y$ direction for Celgard 2500, which shows that the pores are highly elongated; the c-PSD method alone does not reveal this. To aid the visualisation of the elongated slit-like pores, 3D renderings showing the pore phase as solid are presented as inset figures in Fig. 6. In general, Celgard 2500 is shown to have significantly larger pores than Celgard 2325. 
a

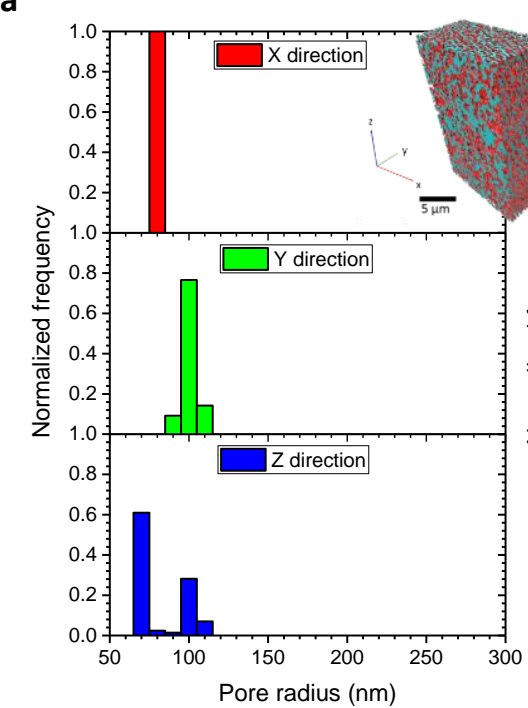

b

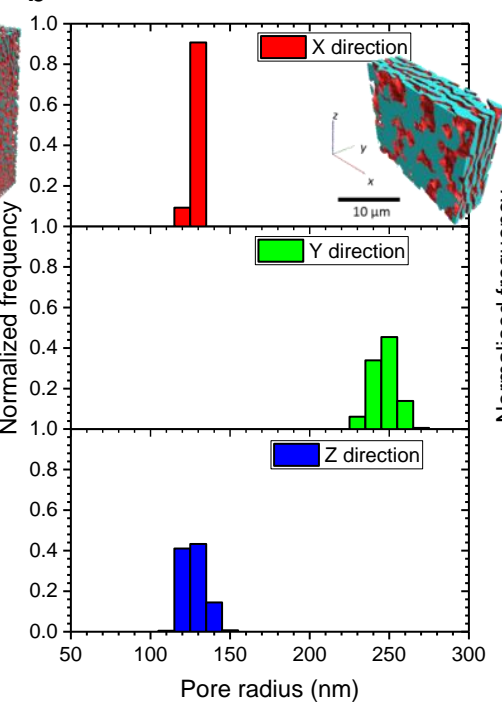

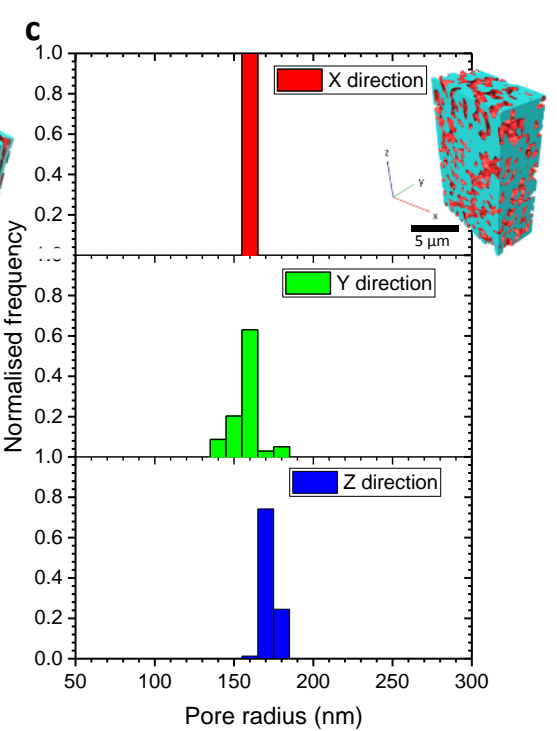

Fig. 6. Normalised frequency plots of the mean pore size calculated by a stereological approach in the $X, Y$ and $Z$ directions for a) Celgard 2325, b) Celgard 2500, and c) the MTI ceramic-coated membrane. Inset: Volume renderings of the three separators showing the pore phase as solid (blue). The 3D renderings highlight the differences in pore size and shape in the each of the axis directions.

\subsection{Tortuosity factor measurement}

As mentioned above, in general it is suspected that a high tortuosity factor is favourable for improving the safety of the cell by providing increased resistance to dendrite growth between electrodes, whereas a low tortuosity factor is favourable for high rate applications, where the resistance to ion transport is low. However, it should be noted that as there is still uncertainty surrounding the formation and growth of lithium dendrites $[49,50]$, the exact link between tortuosity factor and dendrite resistance remains unclear. To determine the tortuosity factor of the separator samples, a finite element simulation of a scalar diffusion parameter $[19,51]$ was used. A representative volume element (RVE) analysis of tortuosity factor (Fig. 7) shows that the volume used to determine the tortuosity factor for each of the samples did not change significantly with sample size, and is therefore considered representative of the bulk material. As seen in Fig. 7a, the MTI ceramic-coated separator has the highest tortuosity factor, followed by Celgard 2325 and Celgard 2500. However, the bulk measurements do not provide any information on the role of the individual layers in ionic transport. The tortuosity factor of Celgard 2325 was considered for four different volumes: one volume consisting of the full tri-layer sample (Fig. 7a), and three more volumes consisting of each of the three layers (PP, PE and PP) (Fig. 7b) separately. Similarly, the tortuosity factors of the three layers of the MTI ceramic-coated membrane (ceramic, PE and ceramic) were assessed separately (Fig. 7c). 

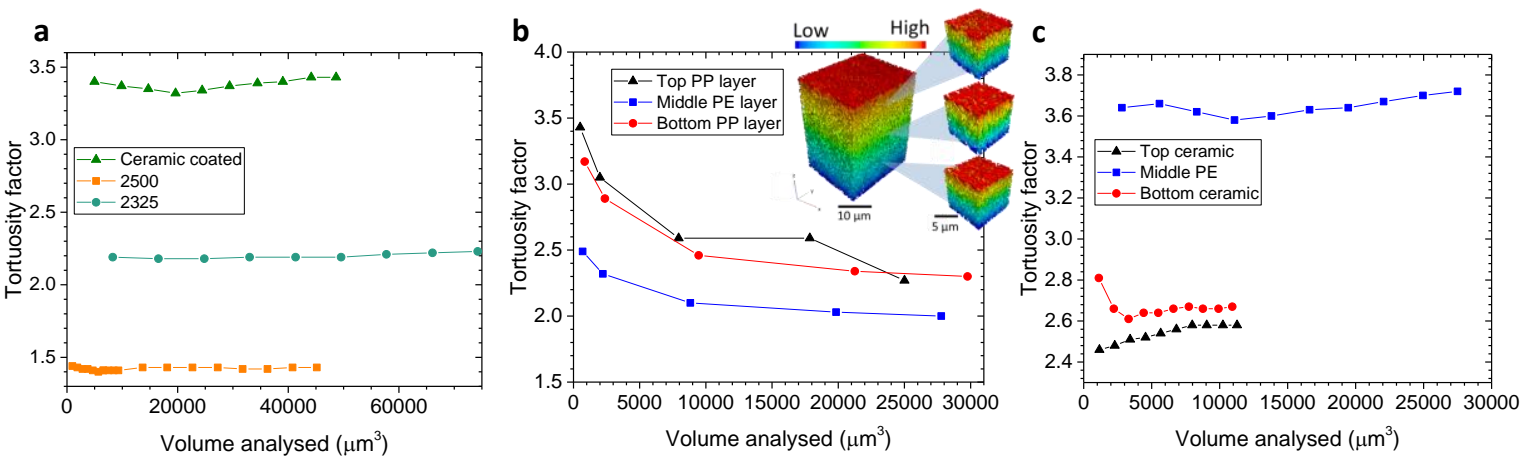

Fig. 7. a) RVE analysis for each of the separator samples showing that the full volumes of Celgard 2325, Celgard 2500 and the MTI ceramic-coated separator provide representative values for the tortuosity factors of the bulk materials. b) RVE analysis of the tortuosity factor for the three individual layers (PP|PE|PP) of Celgard 2325. Inset: 3D representation of the output volumes from the scalar diffusion simulations of each of the three layers. c) RVE analysis of the individual layers from the MTI ceramic-coated separator.

The tortuosity factor values extracted from the full volumes shown in Fig. 7 are presented in Table 2. According to Equation 1, the membrane transport resistance is proportional to the tortuosity factor $(\tau)$, the thickness of the membrane $(I)$, and inversely proportional to the membrane porosity $(\varepsilon)$. The resistance factor $(\boldsymbol{t} / \mathrm{\varepsilon})$ is therefore included in Table 2 to identify the contribution of the individual layers to the ionic transport resistance. Upon comparison with other studies [13, 12, 45], the tortuosity values determined here seem relatively low. However, as discussed by Holzer et al. [26] and Tjaden et al. [51], the tortuosity values are highly dependent on the measurement technique used; here, we use a finite difference diffusion model which does not capture all factors that contribute to the resistance to ion transport, such as the dielectric constant of the electrolyte and the temperature dependence. Additionally, the tortuosity measurements are carried out on the segmented reconstruction of the polymer microstructure, which as previously mentioned, is not expected to have captured the intricate detail below the resolution of the scan.

The deviation between experimentally determined tortuosity values and those found here might also be a result of the limited resolution of the imaging technique itself. For example, the uniaxial stretching during manufacturing leaves nano-fibrils, which bridge the 'slit-like' pores shown above [5]. The presence of the nano-fibrils would certainly hinder ion transport through the membrane, which would appear as an increased tortuosity in the experimental data; however, the nano-fibrils could not be identified using the current imaging approach. Hence, although quantitative, the values provided in Table 2 should be considered with the caveat of the aforementioned systematic limitation of resolution. 
Table 2. Summary of separator properties as determined via X-ray microscopy, where $\varepsilon$ represents porosity, $\tau$ represents the tortuosity factor, $l$ is the thickness of the membrane, and $\tau \mathrm{l} / \varepsilon$ is the resistance factor.

\begin{tabular}{lcccc}
\hline \hline Sample & $\boldsymbol{\varepsilon}$ & $\boldsymbol{\tau}$ & $\begin{array}{c}\boldsymbol{l} \\
(\mu \mathrm{m})\end{array}$ & $\begin{array}{c}\frac{\boldsymbol{\tau} \boldsymbol{\boldsymbol { \varepsilon }}}{\boldsymbol{\varepsilon}} \\
(\mu \mathrm{m})\end{array}$ \\
\hline Celgard 2325 & $\mathbf{0 . 4 1}$ & $\mathbf{2 . 2 3}$ & $\mathbf{2 5}$ & $\mathbf{1 3 6}$ \\
\hline - Celgard 2325 PP top & 0.39 & 2.27 & 8.3 & 48.5 \\
\hline - Celgard 2325 PE & 0.44 & 2 & 8.3 & 37.9 \\
\hline - Celgard 2325 PP bottom & 0.39 & 2.30 & 8.3 & 49.1 \\
\hline Celgard 2500 & $\mathbf{0 . 5 3}$ & $\mathbf{1 . 4 3}$ & $\mathbf{2 5}$ & $\mathbf{6 7 . 5}$ \\
\hline MTI ceramic-coated & $\mathbf{0 . 3 8}$ & $\mathbf{3 . 4 3}$ & $\mathbf{1 6}$ & $\mathbf{1 4 4 . 3}$ \\
\hline - Top ceramic & 0.40 & 2.58 & 2 & 12.9 \\
\hline - Middle PE & 0.36 & 3.72 & 12 & 123.9 \\
\hline - Bottom ceramic & 0.39 & 2.67 & 2 & 13.7 \\
\hline \hline
\end{tabular}

According the data in Table 2, the PP layers in tri-layer Celgard 2325 contribute more per unit membrane thickness to the ionic transport resistance than the PE layer, which emphasises the role of the PP layers in the performance and safety of the cell. For example, an increase in the thickness of the PP layer may contribute more to help mitigate the risk of dendrite growth than an increase in the thickness of the PE layer. In an effort to minimise volume and weight, the primary function of the PE layer might only be to block the pores of the membrane upon shutdown at high temperatures, while the PP layers contribute most to preventing dendrite growth between electrodes. Table 2 also shows that the resistance of the monolayer Celgard 2500 to ion transport is half that of Celgard 2325. The high porosity and low tortuosity values associated with Celgard 2500 make the separator suitable for high-rate applications; whereas the tri-layer design of Celgard 2325 has a relatively high tortuosity and low porosity, which is found to be mostly influenced by the PP layers. The high tortuosity, low porosity and small pore size may improve the safety of the membrane, but would increase its electrical resistance.

A relatively high tortuosity and high resistance to diffusive flux was determined for the MTI ceramiccoated separator. The PE layer exhibits the greatest resistance per unit thickness compared to its counterparts in the Celgard samples, which were manufactured using a dry process. The ceramic layers are shown to have little effect on the overall tortuosity of the membrane, but have been shown to improve the thermal and mechanical properties $[52,53]$, as well as the wettability of polymer separators $[54,55]$. Hence, the combination of high tensile strength resulting from its isotropic structure, high tortuosity, and the presence of the ceramic layer $[52,53]$ would help mitigate the risks associated overheating, dendrite growth and electrode displacement [56, 57], making this separator favourable for safety critical applications. 


\section{Conclusions}

Tortuosity factor, porosity and pore size distribution are among the most important properties to understand when discussing the performance of separator materials. Here, they have each been quantified for three commercial separator membranes via a combination of phase contrast nanoscale X-ray microscopy and image analysis on small but representative volumes. This image-based analysis overcomes some of the limitations of experimental measurement techniques, which are necessarily based on bulk measurements. For example, here we present the possibility of determining variations in porosity and tortuosity factor between individual layers in more complex tri-layer membranes, which cannot be achieved using conventional methods. This has provided new insights into the role of the individual layers within multilayer membranes in the rate capabilities and safety of commercial cells. However, this X-ray imaging approach has limitations such as spatial resolution, and the lack of understanding and quantification of relevant parameters that influence safety and performance. Nevertheless, the results in this study demonstrate that phase contrast Xray microscopy and image-based quantification provide an effective means of understanding the complex pore structure of separator membranes. This technique could be extended to investigate via in-situ tomography techniques, the changes in separator pore structure at elevated temperature and applied strain, leading to a greater understanding of failure mechanisms, particularly if the effect on individual layers within the membranes can be distinguished.

\section{Acknowledgements}

The authors gratefully acknowledge funding from the Engineering and Physical Sciences Research Council (EPSRC), the Royal Academy of Engineering and the National Physical Laboratory (NPL).

\section{References}

[1] C.J. Orendorff, The Electrochemical Society Interface, 21 (2012) 61-65.

[2] E.P. Roth, D.H. Doughty, D.L. Pile, Journal of Power Sources, 174 (2007) 579-583.

[3] Y. Lee, J. Park, H. Jeon, D. Yeon, B.-H. Kim, K.Y. Cho, M.-H. Ryou, Y.M. Lee, Journal of Power Sources, 325 (2016) 732-738.

[4] X. Huang, Journal of Power Sources, 323 (2016) 17-22.

[5] P. Arora, Z. Zhang, Chemical Reviews, 104 (2004) 4419-4462.

[6] B. Münch, L. Holzer, Journal of the American Ceramic Society, 91 (2008) 4059-4067.

[7] S. Diamond, Cement and Concrete Research, 30 (2000) 1517-1525.

[8] H. Giesche, Particle \& Particle Systems Characterization, 23 (2006) 9-19.

[9] E.W. Washburn, Physical Review, 17 (1921) 273-283.

[10] E.W. Washburn, Proceedings of the National Academy of Sciences of the United States of America, 7 (1921) 115-116.

[11] J. Cannarella, C.B. Arnold, Journal of Power Sources, 226 (2013) 149-155.

[12] I.V. Thorat, D.E. Stephenson, N.A. Zacharias, K. Zaghib, J.N. Harb, D.R. Wheeler, Journal of Power Sources, 188 (2009) 592-600.

[13] B. Tjaden, S.J. Cooper, D.J.L. Brett, D. Kramer, P.R. Shearing, Current Opinion in Chemical Engineering, 12 (2016) 44-51.

[14] R.B. MacMullin, G.A. Muccini, AlChE Journal, 2 (1956) 393-403.

[15] G. Venugopal, J. Moore, J. Howard, S. Pendalwar, Journal of Power Sources, 77 (1999) 34-41. 
[16] M.F. Lagadec, M. Ebner, R. Zahn, V. Wood, Journal of The Electrochemical Society, 163 (2016) A992-A994.

[17] E. Maire, P. Withers, International Materials Reviews, 59 (2014) 1-43.

[18] S.R. Stock, International Materials Reviews, 53 (2008) 129-181.

[19] S.J. Cooper, D.S. Eastwood, J. Gelb, G. Damblanc, D.J.L. Brett, R.S. Bradley, P.J. Withers, P.D. Lee, A.J. Marquis, N.P. Brandon, P.R. Shearing, Journal of Power Sources, 247 (2014) 1033-1039.

[20] M. Ebner, D.-W. Chung, R.E. García, V. Wood, Advanced Energy Materials, 4 (2014).

[21] M. Ebner, V. Wood, Journal of The Electrochemical Society, 162 (2015) A3064-A3070.

[22] P.R. Shearing, L.E. Howard, P.S. Jørgensen, N.P. Brandon, S.J. Harris, Electrochemistry Communications, 12 (2010) 374-377.

[23] P.R. Shearing, N.P. Brandon, J. Gelb, R. Bradley, P.J. Withers, A.J. Marquis, S. Cooper, S.J. Harris, Journal of The Electrochemical Society, 159 (2012) A1023-A1027.

[24] Y.-c.K. Chen-Wiegart, R. DeMike, C. Erdonmez, K. Thornton, S.A. Barnett, J. Wang, Journal of Power Sources, 249 (2014) 349-356.

[25] C. Tötzke, G. Gaiselmann, M. Osenberg, J. Bohner, T. Arlt, H. Markötter, A. Hilger, F. Wieder, A. Kupsch, B.R. Müller, M.P. Hentschel, J. Banhart, V. Schmidt, W. Lehnert, I. Manke, Journal of Power Sources, 253 (2014) 123-131.

[26] L. Holzer, D. Wiedenmann, B. Münch, L. Keller, M. Prestat, P. Gasser, I. Robertson, B. Grobéty, Journal of Materials Science, 48 (2012) 2934-2952.

[27] M. Ebner, F. Geldmacher, F. Marone, M. Stampanoni, V. Wood, Advanced Energy Materials, 3 (2013) 845-850.

[28] D.-W. Chung, P.R. Shearing, N.P. Brandon, S.J. Harris, R.E. García, Journal of The Electrochemical Society, 161 (2014) A422-A430.

[29] B. Vijayaraghavan, D.R. Ely, Y.-M. Chiang, R. García-García, R.E. García, Journal of The Electrochemical Society, 159 (2012) A548-A552.

[30] C. Holzner, M. Feser, S. Vogt, B. Hornberger, S.B. Baines, C. Jacobsen, Nat Phys, 6 (2010) 883887.

[31] Celgard 2325. http://www.ldcgm.com/Celgard/CELGARD-2400.pdf (accessed 04/May/16).

[32] Celgard 2500. http://www.Idcgm.com/Celgard/CELGARD-4550.pdf (accessed 04/May/16).

[33] MTI ceramic coated membrane. http://www.mtixtl.com/CeramicCoatedMembraneforLiionBatteryRandD-EQ-bsf-0016-500A.aspx (accessed 04/May/16).

[34] H.E. Exner, Image Anal Stereol, 23 (2004), 73-82.

[35] J.C. Russ, Manual Methods, in: Practical Stereology, Plenum Press, New York, 1986, pp. 42,43.

[36] V. Deimede, C. Elmasides, Energy Technology, 3 (2015) 453-468.

[37] G. Knauf, M. Doshi, Institute of Paper Chemistry, (1986).

[38] C.F. Berg, Transport in Porous Media, 103 (2014).

[39] K.M. Abraham, Electrochimica Acta, 38 (1993) 1233-1248.

[40] D.S. Eastwood, R.S. Bradley, F. Tariq, S.J. Cooper, O.O. Taiwo, J. Gelb, A. Merkle, D.J.L. Brett, N.P. Brandon, P.J. Withers, P.D. Lee, P.R. Shearing, Nuclear Instruments and Methods in Physics Research Section B: Beam Interactions with Materials and Atoms, 324 (2014) 118-123.

[41] K.J. Kim, Y.H. Kim, J.H. Song, Y.N. Jo, J.-S. Kim, Y.-J. Kim, Journal of Power Sources, 195 (2010) 6075-6080.

[42] T. Coffey, S.G. Urquhart, H. Ade, Journal of Electron Spectroscopy and Related Phenomena, 122 (2002) 65-78.

[43] O.O. Taiwo, D.P. Finegan, D.S. Eastwood, J.L. Fife, L.D. Brown, J.A. Darr, P.D. Lee, D.J.L. Brett, P.R. Shearing, Journal of Microscopy, 00 (2016), 1-13.

[44] C. Peabody, C.B. Arnold, Journal of Power Sources, 196 (2011) 8147-8153.

[45] D. Miranda, C.M. Costa, A.M. Almeida, S. Lanceros-Méndez, Solid State Ionics, 278 (2015) 7884.

[46] H. Lee, M. Yanilmaz, O. Toprakci, K. Fu, X. Zhang, Energy \& Environmental Science, 7 (2014) 3857-3886. 
[47] S.J. Cooper, TauFactor. www.sourceforge.net/projects/taufactor/ (accessed 03/May/2016).

[48] S.J. Cooper, Quantifying the Transport Properties of Solid Oxide Fuel Cell Electrodes (Doctoral dissertation), 2015. Retrieved from http://ethos.bl.uk/OrderDetails.do?uin=uk.bl.ethos.684318.

[49] C.T. Love, O.A. Baturina, K.E. Swider-Lyons, ECS Electrochemistry Letters, 4 (2015) A24-A27.

[50] K.J. Harry, D.T. Hallinan, D.Y. Parkinson, A.A. MacDowell, N.P. Balsara, Nat Mater, 13 (2014) 6973.

[51] B. Tjaden, J. Lane, P.J. Withers, R.S. Bradley, D.J.L. Brett, P.R. Shearing, Solid State lonics, 288 (2016) 315-321.

[52] X. Zhu, X. Jiang, X. Ai, H. Yang, Y. Cao, ACS Applied Materials \& Interfaces, 7 (2015) 2411924126.

[53] H. Jeon, D. Yeon, T. Lee, J. Park, M.-H. Ryou, Y.M. Lee, Journal of Power Sources, 315 (2016) 161-168.

[54] D. Fu, B. Luan, S. Argue, M.N. Bureau, I.J. Davidson, Journal of Power Sources, 206 (2012) 325333.

[55] K. Prasanna, C.-S. Kim, C.W. Lee, Materials Chemistry and Physics, 146 (2014) 545-550. [56] D.P. Finegan, M. Scheel, J.B. Robinson, B. Tjaden, I. Hunt, T.J. Mason, J. Millichamp, M. Di Michiel, G.J. Offer, G. Hinds, D.J.L. Brett, P.R. Shearing, Nat Communications, 6 (2015).

[57] D.P. Finegan, E. Tudisco, M. Scheel, J.B. Robinson, O.O. Taiwo, D.S. Eastwood, P.D. Lee, M. Di Michiel, B. Bay, S.A. Hall, G. Hinds, D.J.L. Brett, P.R. Shearing, Advanced Science, 3 (2016). 